\title{
Heparin modified polyethylene glycol
}

\section{microparticle aggregates for focal cancer}

\section{chemotherapy}

F. Philipp Seib ${ }^{1,2,3, *, *}$, Mikhail Tsurkan ${ }^{2,+, *}$, Uwe Freudenberg ${ }^{2}$, David L. Kaplan ${ }^{3}$, Carsten Werner $^{2}$

(1) Strathclyde Institute of Pharmacy and Biomedical Sciences, University of Strathclyde, 161 Cathedral Street, Glasgow G4 0RE, United Kingdom.

(2) Leibniz-Institut für Polymerforschung Dresden e.V., Max Bergmann Centre for Biomaterials, Hohe Str. 6, Dresden 01069, Germany

(3) Tufts University, Department of Biomedical Engineering, 4 Colby Street Medford, MA 02155, USA

* Corresponding authors:

F. Philipp Seib: Tel.: +44 141548 2510; emails: philipp.seib@strath.ac.uk or philipp.seib@seiblab.com

Mikhail Tsurkan; Tel.: +49 3514658 644; email: tsurkan@ipfdd.de

Total number of figures: 5 


\title{
Supplementary figures: 2
}

Disclosure statement: The authors have no competing financial interests

\begin{abstract}
Focal cancer therapy can improve clinical outcomes. Here, we evaluated injectable heparin-containing hydrogel material loaded with doxorubicin as a focal breast cancer therapy. We utilized a binary heparin/polyethylene glycol (PEG) hydrogel that was processed post synthesis into hydrogel microparticle aggregates to yield a readily injectable hydrogel. When loaded with doxorubicin, the injectable hydrogel microparticle aggregates had excellent short- and long-term anticancer activity against human breast cancer cells in vitro. Efficacy as a focal anticancer therapy was also evaluated in vivo by local injection of the doxorubicin-loaded PEG-heparin hydrogel microparticle aggregates into mice with established human orthotopic breast tumours. Animals showed significant antitumour responses by reduction in both primary tumour growth and metastasis when compared to animals which received the equivalent doxorubicin dose via an intravenous bolus injection. Overall, PEG-heparin hydrogel microparticle aggregates are emerging as a potential anticancer drug delivery system for focal therapy.
\end{abstract}

Key words: breast cancer, hydrogel, microparticle drug delivery 


\section{Introduction}

Focal chemotherapy is routinely used for a number of malignancies. For example, bladder cancer is often treated with intravesicular chemotherapy, while high-grade malignant glioma is treated using carmustine-containing synthetic copolymer wafers (Gliadel Wafer). In the latter case, after tumour resection, the gliadel wafers are placed into the tumour bed, and more than $15 \%$ of the dose remains at the local site at 7 days post application, thereby increasing brain concentrations more than 113-fold when compared to systemic delivery of carmustine ${ }^{1}$.

The standard clinical practice for early stage breast cancer is typically surgical resection of the primary breast tumour, followed by localized radiotherapy to the affected breast ${ }^{2}$. This treatment strategy reduces ipsilateral tumour recurrence and enhances overall survival ${ }^{3-4}$. Therefore, locoregional control is necessary to improve long-term clinical outcomes. Notably, in this patient population, systemic chemotherapy provides limited control over the locoregional disease ${ }^{2}$. The finding that dual therapy consisting of tumour resection and local irradiation significantly improves treatment success indicates that direct application of chemotherapy to the primary tumour site might improve current clinical outcomes ${ }^{3-4}$. Furthermore, target radiotherapy applied to the tumour bed at the time of surgery has produced encouraging results in low risk women over the age of 45 (TARGIT-A trial) ${ }^{5}$ and is now being tested in high risk women (TARGIT-B trial). However, for low risk ductal carcinoma in situ (DCIS) the current surgical intervention is being challenged and thus a more conservative treatment strategy for DCIS might emerge in the future (Phase III LORIS clinical trial, UK Clinical Research Network ID 16736 and COMET study in the United States) ${ }^{6}$. 
Synthetic polymers are widely used for drug delivery applications; for example, poly(2hydroxyethyl methacrylate) (PHEMA) was used in first generation hydrogels. Since then several synthetic polymers, such as polyesters, polyphosphazene and poly(ethylene glycol) (PEG), have been developed ${ }^{7}$. Many of these polymers are in pre-clinical development for controlled drug delivery applications, including anticancer drug delivery ${ }^{8}$. Of these, PEG has an excellent clinical track record despite being non-biodegradable; careful selection of molecular weight, dose and route of administration have contributed to its clinical safety record. Based on the existing clinical experience with PEG, PEG-based hydrogels have been developed to deliver a wide spectrum of payloads including chemotherapeutic agents (e.g. doxorubicin, paclitaxel) ${ }^{9}$, therapeutic proteins (e.g. befaziumab, exenatide) ${ }^{10-11}$ and precision medicines (e.g. crizotinib) ${ }^{12}$. Payload delivery can be achieved through polymer network degradation, drug diffusion or a combination of both these processes. The use of "bioresponsive" PEG polymers ensures network degradation, which in turn regulates drug release and ultimately leads to polymer elimination. For example, PEG-based hydrogels have been developed with matrix metalloprotease (MPP)sensitive peptide linkers that permit stimulus-mediated carrier degradation and subsequent polymer elimination (reviewed by ${ }^{8}$ ). A number of biopolymer-based systems are also in preclinical development for the focal cancer therapy. For example, we have developed silk-based films and self-assembling silk hydrogels for the focal delivery of chemotherapeutic drugs and precision medicines ${ }^{13-14}$.

Biopolymers for drug delivery are typically expected to elicit no biological response; however, many biopolymers have intrinsic biological properties. For example, heparin in its freely diffusible as well as conjugated form binds to the enzyme inhibitor antithrombin III to induce a 
conformational change of the protein and ultimately inhibit the coagulation cascade ${ }^{15}$. An association between venous thromboembolism and cancer has long been recognised; thus, heparin-based prophylaxis and treatment are routine clinical practices ${ }^{16}$. Furthermore experimental ${ }^{16-17}$ and clinical data ${ }^{18}$ suggest that freely diffusible heparin displays an array of anticancer properties; therefore, heparin use in cancer patients goes beyond the prevention of venous thromboembolism. Furthermore, heparin is a substrate for heparanase which is overexpressed in tumours ${ }^{19}$, and therefore might facilitate on-demand lead drug release. Clinical experience with heparin spans more than 80 years, including various routes of administration (e.g., subcutaneously or by intravenous injections). Therefore, heparin is emerging as a valuable building block for drug delivery systems due to its biological properties. Heparin-containing hydrogels have been evaluated in tissue/disease specific niche models (e.g. ${ }^{20-21}$ ) and as an advanced coagulation coating ${ }^{15}$. The present study pioneers injectable heparin-containing hydrogels as a prospective anticancer therapy.

The aim of the current study was to develop and test semi-synthetic hydrogel-based microparticle aggregates for focal cancer therapy. We designed microparticles using PEGheparin hydrogels; PEG stars (starPEG) with a molecular weight (MW) of 10,000 g/mol were chosen because these polymers permit the formation of hydrogels with a defined $6+/-3 \mathrm{~nm}$ mesh size. A bio-hybrid PEG-heparin hydrogel was fabricated to evaluate the efficacy of heparin hydrogel materials as drug-binding reservoirs and the advantages of local over the systemic delivery of doxorubicin in preventing cancer progression. 


\section{Experimental}

Reagents for PEG-heparin hydrogels

Heparin (MW 14,000 g/mol) was purchased from Calbiochem (Merck, Darmstadt, Germany) and four-armed PEG stars (starPEG; Mn $10 \times 10^{3}$; polydispersity index 1.09) were obtained from PolymerSource (Montreal, Canada). N-hydroxysulfosuccinimide (NHS) was purchased from Sigma-Aldrich (Munich, Germany). Doxorubicin hydrochloride salt was purchased from LC Laboratories (Woburn, MA, USA). All solvents and N-(3-dimethylaminopropyl)-N'ethylcarbodiimide hydrochloride (EDC hydrochloride) for hydrogel synthesis were purchased from IRIS Biotech GmbH (Marktredwitz, Germany). All reagents were used without further purification.

\section{Preparation of PEG-heparin hydrogels}

Hydrogels were prepared as previously described ${ }^{22}$. Briefly, a two-fold excess of EDChydrochloride and a stoichiometric amount of NHS were added to a $\mathrm{pH} 7.0$ solution of heparin in phosphate buffered saline (PBS). The heparin-NHS ester was formed by maintaining the reaction mixture at $4{ }^{\circ} \mathrm{C}$ for $15 \mathrm{~min}$. Next, a stoichiometric amount of starPEG solution in PBS (pH 7.4) was added. The total volume of the reaction mixture was 1 $\mathrm{ml}$ and the total weight of the heparin and starPEG mixture was $11 \% \mathrm{w} / \mathrm{w}$. The reagents underwent a solution-gel (solgel) transition in less than $1 \mathrm{~h}$ and was kept overnight at room temperature for completion of the reaction. The hydrogel was then swollen in purified water (Millipore, Billerica, MA, USA), followed by PBS, to remove the side products of the reaction. The swollen hydrogels were then stored in PBS at $4{ }^{\circ} \mathrm{C}$ until use. Hydrogels used for in vitro and in vivo applications were prepared under sterile conditions and reagent solutions 
were sterilized by filtration through $0.2 \mu \mathrm{m}$ filters (polyvinylidene fluoride membrane, Acrodisc LC 13mm, PALL Life Science, Port Washington, NY, USA).

\section{Preparation of PEG-heparin hydrogel microparticles}

Injectable hydrogel microparticles were prepared as previously described ${ }^{23}$. Briefly, $1 \mathrm{ml}$ of hydrogel was minced using a sterile spatula, collected in a $1 \mathrm{ml}$ syringe and repeatedly extruded through a $0.9 \mathrm{~mm}$ needle $(20 \mathrm{G})$ until a homogenised hydrogel was obtained. The procedure was then repeated with a $0.45 \mathrm{~mm}$ needle $(26 \mathrm{G})$ yielding $25-50 \mu \mathrm{m}$ particles. These hydrogel microparticles were washed with $1 \mathrm{ml}$ purified water by vortexing, centrifuged at $12,110 \mathrm{~g}$ for $2 \mathrm{~min}$ at room temperature and the supernatant was aspirated. This washing procedure was repeated twice more with purified water followed by four PBS washes. As the final step, the microparticles were centrifuged at 12,110 g for 6 min and any released liquid was removed. The resulting hydrogel microparticle aggregates were stored at $4^{\circ} \mathrm{C}$ until use. Microparticle aggregates for in vitro and in vivo applications were prepared under sterile conditions. After centrifugation, the formed hydrogel microparticle aggregate was uploaded into sterile syringes and stored at $4^{\circ} \mathrm{C}$.

\section{Loading of the PEG-heparin hydrogel microparticle aggregates with doxorubicin}

A $1 \mathrm{ml}$ hydrogel microparticle volume was prepared under sterile conditions, as detailed above. Next, $50 \mu 1$ of sterile water containing doxorubicin at either 40, 200, 400 or $800 \mu \mathrm{g}$ were added. Samples were extensively vortexed for $15 \mathrm{~min}$ and hydrogel microparticles were left to equilibrate at room temperature for $60 \mathrm{~min}$. The mixing-equilibration procedure was repeated until hydrogel microparticle aggregates showed an even drug distribution. Next, the 
hydrogel microparticle aggregates were centrifuged at $12,110 \mathrm{~g}$ for $15 \mathrm{~min}$ and the supernatant was removed. Doxorubicin-loaded PEG-heparin hydrogel microparticle aggregates were filled into $1 \mathrm{ml}$ syringes, which were then hermetically sealed and stored at $4^{\circ} \mathrm{C}$ until use.

In vitro doxorubicin release from PEG-heparin hydrogel microparticle aggregates

To assess doxorubicin release from $50 \mu \mathrm{l}$ hydrogel microparticle aggregates, samples were incubated with PBS at $37^{\circ} \mathrm{C}$. Cumulative drug release was monitored by removing and replacing the buffer at the indicated time points and measuring doxorubicin-associated UV absorbance at $490 \mathrm{~nm}$ (Beckman Coulter, DU800, USA). PEG-heparin hydrogel microparticle aggregates without doxorubicin were used to establish an absorbance reference value (data not shown). For studies that determined the binding affinity of doxorubicin to the PEG-heparin hydrogel microparticle aggregates drug release was monitored without changes of the release buffer.

In vitro studies breast cancer studies

The breast cancer cell lines MDA-MB-231 and MCF-7 were obtained from ATCC (Manassas, VA, USA). Cells were maintained in a humidified atmosphere of $5 \% \mathrm{CO} 2$ at $37{ }^{\circ} \mathrm{C}$, and cultures were subcultured every 2-3 days. The MDA-MB-231 cells were grown in RPMI 1640 with 10 \%v/v FBS; MCF-7 cells were cultured in DMEM (4.5 g glucose, $110 \mathrm{mg}$ sodium pyruvate) supplemented with $10 \% \mathrm{v} / \mathrm{v}$ FBS and $10 \mu \mathrm{g} / \mathrm{ml}$ insulin. In vitro studies, including the disease relapse assay, were based on protocols developed previously ${ }^{24}$. Briefly, cells were plated at a density of $2 \times 10^{4}$ cells $/ \mathrm{cm}^{2}$ in the lower chamber of Transwells (Corning, New York, USA). 
Cultures were allowed to recover for $24 \mathrm{~h}$. Next, PEG-heparin hydrogel microparticle aggregates $(100 \mu \mathrm{l})$ were added to the chamber inserts $(0.4 \mu \mathrm{m}$ pore size). As a control, free doxorubicin at doses of 4, 20, 40 and $80 \mu \mathrm{g}$ was added to control wells to represent the total drug loading. For endpoint studies, cell viability was determined after a $72 \mathrm{~h}$ exposure time with (3-(4,5dimethylthiazol-2-yl)-2,5-diphenyltetrazolium bromide (MTT) as a substrate. Following a $5 \mathrm{~h}$ incubation period, formazan was solubilized with dimethylsulfoxide, and the absorbance was measured at $560 \mathrm{~nm}$.

For long-term studies, analogous studies were performed using AlamarBlue (Invitrogen, Grand Island, NY, USA) instead of MTT and an initial seeding density of $1 \times 10^{4}$ cells $/ \mathrm{cm}^{2}$. At the indicated times, AlamarBlue was added to the culture medium, and cell viability was measured after a $4 \mathrm{~h}$ incubation period by monitoring fluorescence. Next, the medium was replaced with fresh culture medium, and cultures were continued. Disease relapse was mimicked by re-seeding the culture inserts at day 6 with $1 \times 10^{4} \mathrm{cells} / \mathrm{cm}^{2}$, and viability was monitored as detailed above. The only culture that was not re-seeded was the control group because it had reached confluency by this time.

\section{Orthotopic human xenografts}

All in vivo studies were approved by the Institutional Animal Care and Use Committee (Protocol B2010-101), and animals were maintained under the guidelines established by the National Institute of Health and Tufts University. Tumour xenografts were induced using MDA-MB-231derived tumour cells that metastasized following orthotopic injection into mice; the tumours 
carried the firefly luciferase gene to permit in vivo bioluminescence imaging ${ }^{25}$. In vivo studies were based on protocols developed previously ${ }^{24}$. Briefly, female NOD/SCID mice (NOD.CB17$\operatorname{Prkdc}^{\text {scid }} / \mathrm{NcrCrl}$ ) aged 6 to 10 weeks were obtained from Charles River (Wilmington, MA, USA). A total of $5 \times 10^{5}$ cells in $20 \mu$ of Matrigel (BD Biosciences, Bedford, MA, USA) were injected bilaterally into the fat pad. Following tumour induction, mice (group sizes 4 to 5) were randomised and treated at day 12 with $100 \mu$ of PEG-heparin hydrogel microparticle aggregates containing $40 \mu \mathrm{g}$ doxorubicin; these injections were performed using a G26 needle. Mice received bilateral injections close to the tumour sites (i.e., $80 \mu \mathrm{g}$ of doxorubicin/mouse) but were not given an intratumour injection. As a control, the equivalent doxorubicin dose of $80 \mu \mathrm{g}$ was administered in $100 \mu \mathrm{l}$ of PBS via a bolus tail vein injection to the control mice. Disease progression was monitored weekly by tumour cell-associated bioluminescence imaging using the Xenogen IVIS 200 imaging system controlled by the Living Image Software 4.2 (Caliper Life Sciences, Hopkinton USA). At the endpoint of the study, the brain, lung, liver and bones of each mouse were examined for metastasis, and the primary tumours were dissected and weighed. Histology was performed as detailed elsewhere ${ }^{24}$.

\section{Statistical analysis}

Data were analysed using GraphPad Instat 5.0b (GraphPad Software, La Jolla USA). Sample pairs were analysed with the Student's t-test. Multiple samples were evaluated by one-way analysis of variance followed by Bonferroni or Dunnett's post hoc tests to evaluate the statistical differences $(P \leq 0.05)$ among all samples or between samples and controls, respectively. All error bars were standard deviation (SD) unless otherwise stated. An asterisk denotes statistical significance as follows: $* \mathrm{P}<0.05,{ }^{* *} \mathrm{P}<0.01, * * * \mathrm{P}<0.001$. 


\section{Results}

The heparin-modified PEG hydrogels synthesised with a $10 \mathrm{mg} / \mathrm{ml}$ heparin final concentration showed an average pore size of $6+/-0.5 \mathrm{~nm}$ and $13+/-0.5 \mathrm{kPa}$ stiffness ${ }^{21,23}$. Shearing was used to generate microparticles with an average mean diameter of 25-50 $\mu \mathrm{m}$ (Fig. 1a) and these microparticles were readily injectable through a G26 needle. Such particles form stable aggregates post injection and have defined viscoelastic properties; a storage modulus (i.e. stiffness) of $300 \mathrm{~Pa}$ and a loss modulus (i.e. viscous properties) of $90 \mathrm{~Pa}$ as reported previously by us ${ }^{23}$. These particles were loaded with doxorubicin (Fig. 1b); drug loading could be finetuned over a broad concentration range (4 to $80 \mu \mathrm{g}$ doxorubicin/hydrogel) (Supplementary Fig. 1). In vitro cumulative drug release studies showed complete doxorubicin release $(>95 \%)$ within 100 hours for PEG-heparin hydrogel microparticle aggregates with a $4 \mu \mathrm{g}$ doxorubicin loading while 30\% was achieved for $40 \mu \mathrm{g}$ drug loaded carriers (Fig. 2a). Cumulative drug release studies revealed the relationship between doxorubicin release and drug loading. Following an initial transient burst release a zero-order like drug release rate was observed (Fig. 2c and d). These measurements were underpinned by equilibrium binding studies to assess the distribution of doxorubicin in the hydrogel and supernatant (Fig. 2b, Supplementary Fig. 2b, d, f); these measurements were used to calculate the binding and dissociation constants. The PEG-heparin hydrogel microparticle aggregates had an approximate doxorubicin binding constant of $\mathrm{K}_{\mathrm{a}} 5 \times$ $10^{2} \mathrm{M}^{-1}$ and a dissociation constant of $\mathrm{K}_{\mathrm{b}} 2 \times 10^{-3} \mathrm{M}^{-1}$. 


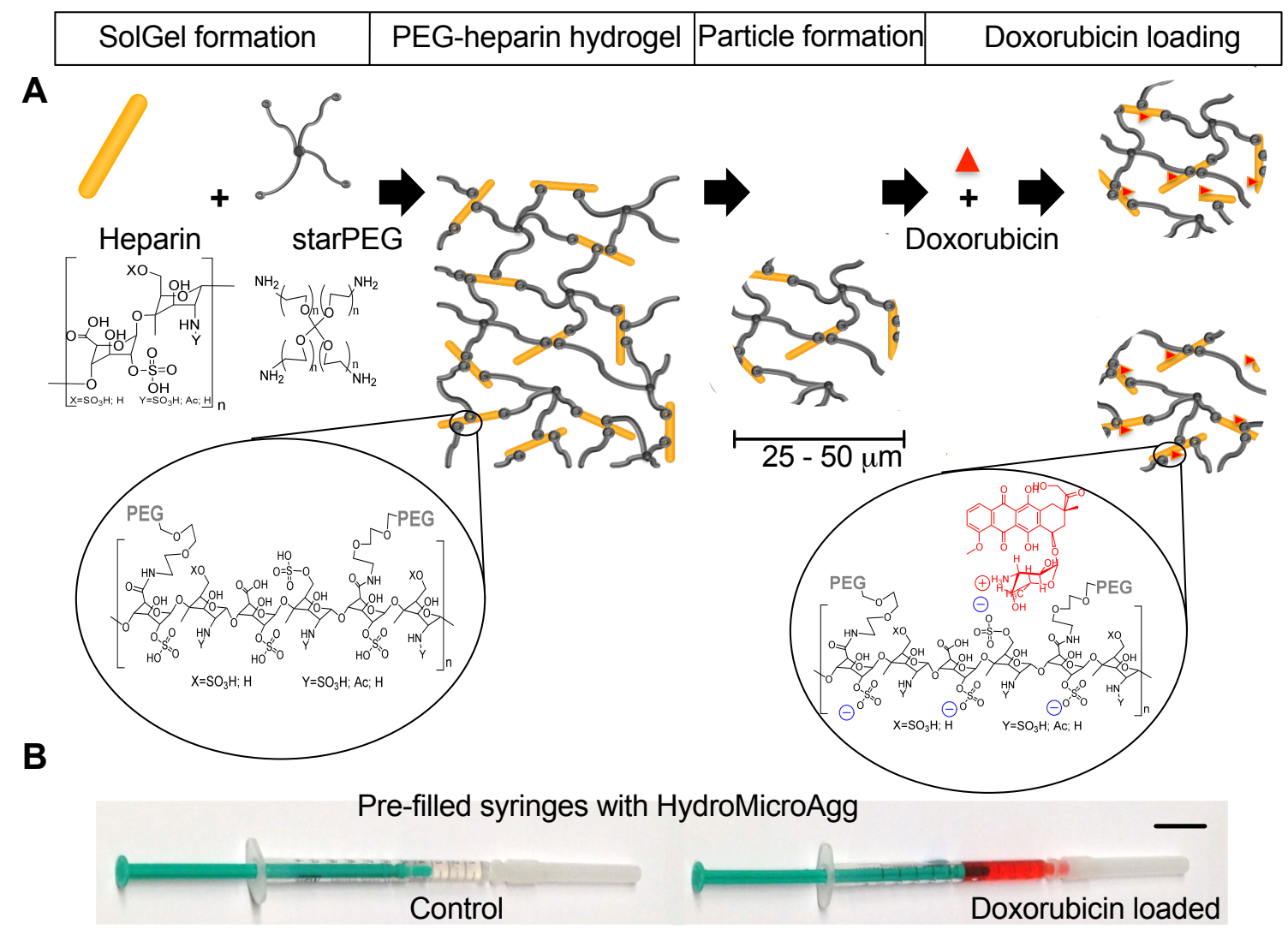

Figure 1. Schematic representation of the manufacture of drug-loaded microparticles. (A) Synthesis of heparin-modified polyethylene glycol (PEG) hydrogels; post synthesis hydrogels are processed into particles and drug loaded. (B) Images of control and doxorubicin-loaded PEGheparin hydrogel microparticle aggregates (HydroMicroAgg) in pre-filled $1 \mathrm{ml}$ syringes for biological application and assessment. 
B
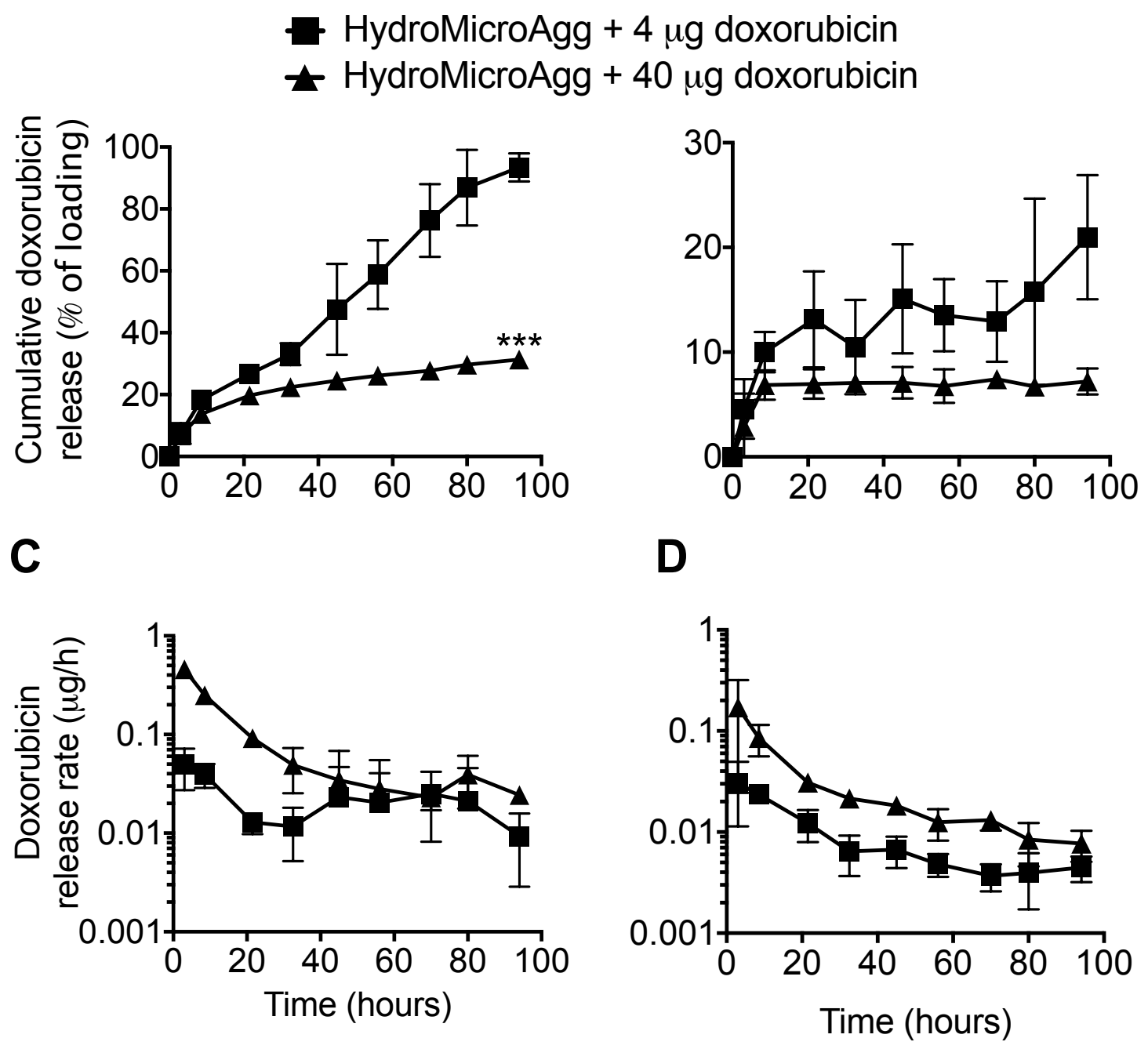

Figure 2. Drug release from PEG-heparin hydrogel microparticle aggregates (HydroMicroAgg). Cumulative doxorubicin release when buffer was (A) replaced with fresh one at each sampling time point or (B) kept throughout the experiment. Corresponding doxorubicin release rates when the release buffer was replaced (C) or (D) kept. Error bars are hidden in the plot-symbol when not visible, $\mathrm{n}=4 ; \pm \mathrm{SD}$ 
The in vitro cytotoxicity of doxorubicin-loaded PEG-heparin hydrogel microparticle aggregates was assessed using human oestrogen-responsive breast cancer cells (MCF-7) and a highly aggressive triple negative breast cancer cell line (MDA-MB-231). Short-term (72h) cytotoxicity assays showed a significant antitumor response for MCF-7 cells (cell viability $<10 \%$ ) across the tested concentration range, with a comparable response for freely diffusible drug and doxorubicin-loaded PEG-heparin hydrogel microparticle aggregates (Fig. 3a). In contrast, MDAMB-231 breast cancer cells showed approximately $30 \%$ cell viability at a $4 \mu \mathrm{g}$ drug dose; this response was independent of the delivery mode (Fig. 3a). At all other drug concentrations, cell viability was $<10 \%$, with no significant differences between freely diffusible drug and doxorubicin-loaded PEG-heparin hydrogel microparticle aggregates (Fig. 3a). We used a breast cancer relapse assay ${ }^{24}$ to examine the full potential of doxorubicin-loaded PEG-heparin hydrogel microparticle aggregates (Fig. 3b). Freely diffusible doxorubicin and doxorubicin-loaded PEGheparin hydrogel microparticle aggregates showed significant cytotoxicity with no significant differences for the first 6 days of the assay (Fig. 3b). However, at day 8, the doxorubicin-loaded injectable PEG-heparin hydrogel microparticle aggregates continued to control breast cancer cell growth of both MCF-7 and MDA-MB-231 cell lines and significantly suppressed cell growth when compared to the diffusible doxorubicin control groups (Fig. 3b). 

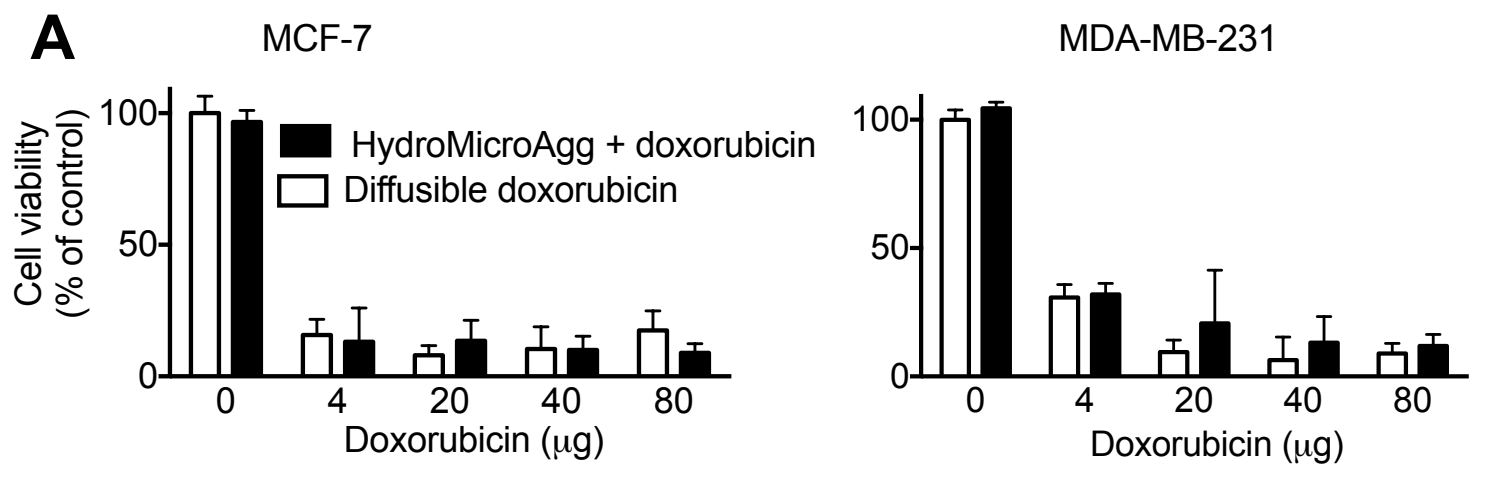

B

$\Delta$ HydroMicroAgg $+40 \mu g$ doxorubicin

- HydroMicroAgg conrol

- HydroMicroAgg $+4 \mu \mathrm{g}$ doxorubicin

$\square$ Diffusible doxorubcin $4 \mu \mathrm{g}$

$\triangle$ Diffusible doxorubicin $40 \mu \mathrm{g}$
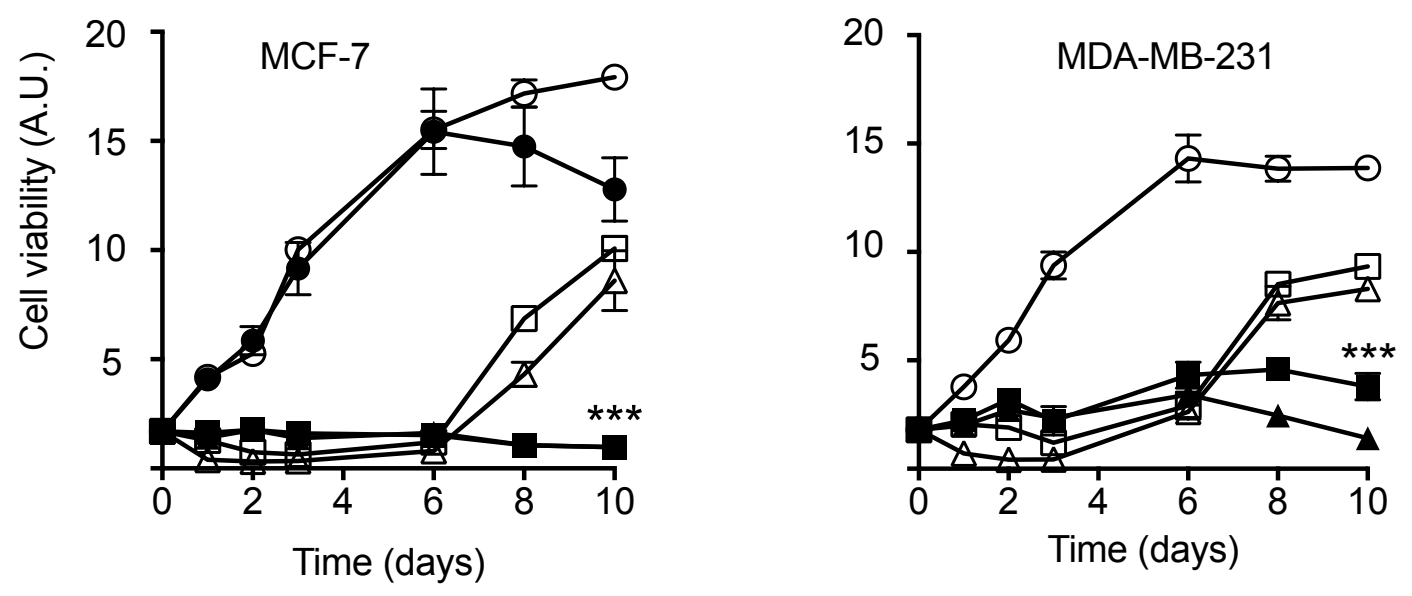

Figure 3. In vitro cytotoxicity of breast cancer cells treated with doxorubicin-loaded PEGheparin hydrogel microparticle aggregates (HydroMicroAgg). (A) Cell viability of MDA-MB231 and MCF7 cells treated for $72 \mathrm{~h}$ with HydroMicroAgg loaded with various amounts of doxorubicin and respective controls with equivalent amounts of diffusible doxorubicin. (B) Long-term cytotoxicity of free doxorubicin and HydroMicroAgg loaded with doxorubicin in MDA-MB-231 and MCF7 breast cancer cells. Culture medium was replaced at the indicated time. With the exception of the controls, all wells were re-seeded with the corresponding breast cancer cells at day 6. Significant differences between diffusible doxorubicin and the other 
doxorubicin treatment groups were determined, followed by Dunnett's multiple comparison post hoc test, ${ }^{* * *} \mathrm{P}<0.0001$; error bars are hidden in the plot-symbol when not visible, $\pm \mathrm{SD} ; \mathrm{n}=4$.

Based on these in vitro study results, we assessed the ability of doxorubicin-loaded PEG-heparin hydrogel microparticle aggregates to control tumour growth in vivo (Fig. 4a). Because triple negative breast cancer poses a real clinical challenge, we tested our delivery system using triple negative MDA-MB-231 orthotopic breast cancer model. Bioluminescence monitoring of animals treated with drug-loaded PEG-heparin hydrogel microparticle aggregates revealed a significantly reduced tumour burden at 3 weeks and all subsequent time points when compared to bolustreated mice (Fig. 4b). Dissection and weighing of tumours from animals treated with drugloaded PEG-heparin hydrogel microparticle aggregates confirmed a significantly reduced tumour burden when compared to bolus-treated animals (Fig. 4 b). Assessment for breast cancer metastasis revealed that animals treated with drug-loaded PEG-heparin hydrogel microparticle aggregates showed a small increase in lung metastasis, whereas metastasis to brain, liver and bone showed a substantial reduction when compared to the bolus-treated animals (Fig. 4 c). 

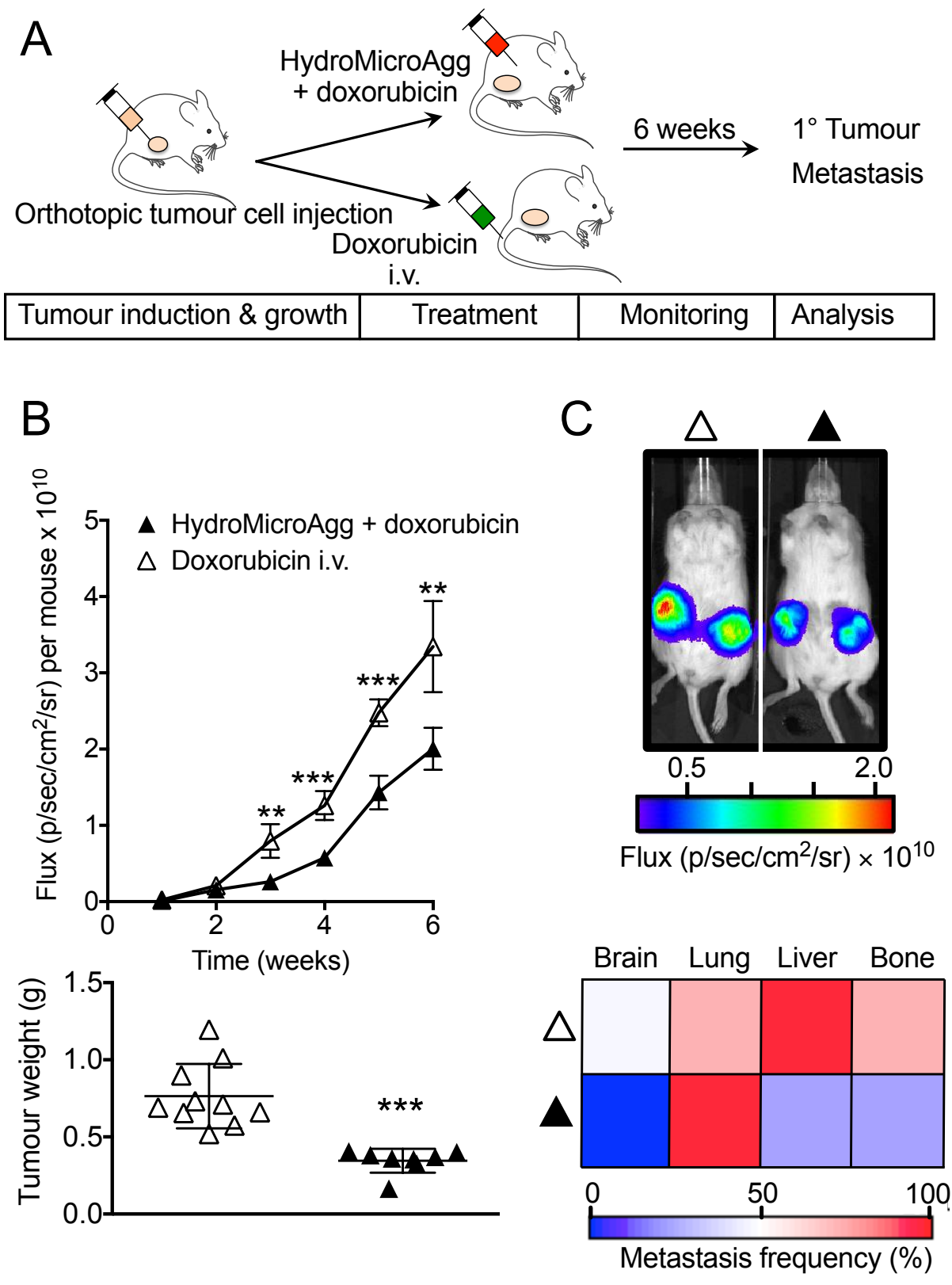

Figure 4. In vivo response of doxorubicin-loaded PEG-heparin hydrogel microparticle aggregates (HydroMicroAgg). (A) Schematic of the experimental approach. (B) Treatment of tumour-bearing mice either with doxorubicin-loaded HydroMicroAgg or the equivalent amount of doxorubicin administered by intravenous bolus dosing. Tumour growth was monitored in vivo by non-invasive, cancer cell-specific bioluminescence imaging and primary tumour weights were assessed the end of the study. (C) Bioluminescence images of mice at week 6 and metastatic 
spread of cancer cells to organs at week 6 . Statistical differences were determined using unpaired T-test, $* * P<0.005, * * * P<0.001 ; \pm \mathrm{SD}$ tumour weights and standard error of the mean for tumour growth; error bars are hidden in the plot-symbol when not visible, $n=5$ doxorubicin i.v., $n=4$ PEG-heparin hydrogel microparticle aggregates.

PEG-heparin hydrogel microparticle aggregates remained within the initial injection site and did not dislocate over the course of the study. PEG-heparin hydrogel microparticle aggregate samples retrieved and subjected to histological examination (Fig. 5) were readily identifiable (Fig. 5 a) and discrete hydrogel microparticles were visible in histological sections (Fig. 5 c). Overall, PEG-heparin hydrogel microparticle aggregates showed a low foreign body response, with minimal cell infiltration and connective tissue encapsulation at both the ventral and dorsal orientation (Fig. 5a-c). 

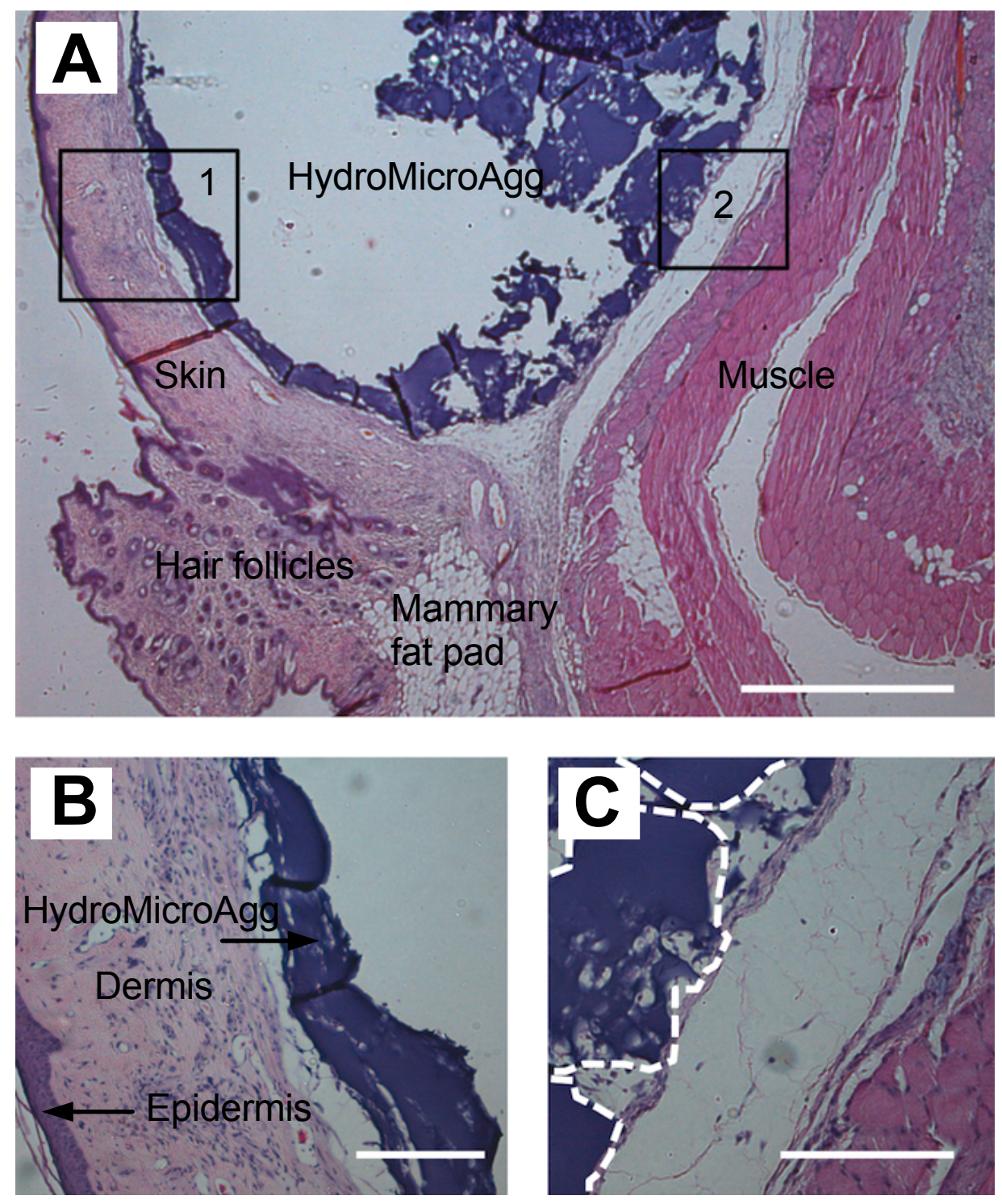

Figure 5. In vivo histocompatibility assessment of PEG-heparin hydrogel microparticle aggregates (HydroMicroAgg). Histological section and H\&E staining of HydroMicroAgg; samples were retrieved from the study detailed in Figure 4. (A) Low magnification image showing HydroMicroAgg (scale bar 1,000 $\mu \mathrm{m}$ ). Higher magnifications for areas 1 and 2 are shown in panel (B) and (C), respectively (all scale bars $250 \mu \mathrm{m}$ ). Dotted lines in panel (C) delineate particle outlines. 


\section{Discussion}

Our aim was to assess heparin-containing polyethylene glycol microparticle aggregates for focal cancer chemotherapy. We selected PEG and heparin as building blocks of our formulation because of the substantial clinical experience with both materials. For example, PEG is a commonly used excipient in pharmaceutical preparations (e.g., Molaxole $($, Movicol®) where several grams of PEG (MW between 3.000 - $6.000 \mathrm{~g} / \mathrm{mol}$ ) are administered orally. Furthermore, PEG is commonly used to modulate the pharmacokinetics of nanomedicines by grafting the polymer to proteins, peptides, aptamers or small molecular weight drugs (reviewed in ${ }^{26-27}$ ). Typically PEG is regarded as a "safe" polymer for a broad spectrum of applications; this is achieved by controlling both the MW and dose.

For hydrogel-based systems intended for subcutaneous administration, PEG induces a minimal foreign body response because it limits nonspecific protein adsorption on the implant surface ${ }^{28}$. Protein adsorption, also known as fouling, is considered to be the first step in the foreign body response $^{29}$. Any implanted material will induce a foreign body response, but PEG-based systems show very low fouling properties ${ }^{28,30}$. This clinical track record for PEG prompted its selection in the present study for the generation of hydrogel microparticles for controlled drug delivery. We wanted to endow these PEG hydrogel with improved drug release capabilities, so we generated heparin-functionalised hydrogels (Fig. 1) by crosslinking the carboxylic acid groups of heparin with the four primary amine groups of starPEG using carbodiimide chemistry (Fig. 1a) 22. The starPEG-heparin hydrogel reported here is stable towards cell-mediated enzymatic degradation ${ }^{31}$. Furthermore, these hydrogels have shown remarkable non-cell-adhesive properties that are comparable to fully synthetic PEG hydrogels ${ }^{32}$. Heparin is particularly well 
suited for the intended application because it has known anticancer activity in its unbound form (i.e. freely diffusible) ${ }^{17}$. Notably, the anticancer activity of heparin conjugated into material has not been described previously, to the best of our knowledge. Our other intention was to explore the possibility that the negative net charge of heparin could facilitate the binding of drugs like doxorubicin, a weakly basic anticancer drug. The physical properties of these PEG-heparin hydrogel-based microparticle aggregates, characterised previously using dynamic mechanical analysis ${ }^{23}$, confirmed that injectable hydrogel aggregates behave as intact hydrogel after injection.

Drug release from PEG-heparin hydrogel microparticle aggregates was dependent on drug loading (Fig. 2, Supplementary Fig. 2). Here, complete drug release was achieved for systems loaded with $4 \mu \mathrm{g}$ of doxorubicin while $30 \%$ drug release was achieved for $40 \mu \mathrm{g}$ drug loaded PEG-heparin hydrogel microparticle aggregates. These data suggest that doxorubicin release was not following a linear, dose dependent release profile. One possible explanation is doxorubicin's ability to $\pi-\pi$ stack that becomes more prominent at higher drug loadings. Calculations of binding and a dissociation constants suggested a complex doxorubicin release mechanism with a significant contribution of the hydrogel on doxorubicin diffusion and release kinetics because the calculated dissociation constant was too high to sustain the experimentally observed long-term drug release kinetics. Next, we examined the in vitro response of our doxorubicin-loaded PEGheparin microparticle aggregates using the oestrogen receptor-positive, hormone-responsive, Her2/neu-negative breast cancer cell line (MCF7) and triple negative breast cancer cells (MDAMB-231) (Fig. 3). All treatment groups showed an excellent response in short-term cytotoxicity assays. However, our breast cancer relapse assay showed that the diffusible doxorubicin 
treatment failed to control outgrowth of breast cancer cells (Fig. 3b). In contrast, our drug-loaded PEG-heparin hydrogel microparticle aggregates controlled breast cancer cell growth over the entire study period. These results strongly suggest that, under these in vitro culture conditions, doxorubicin was released at cytotoxic concentrations over an extended period of time. We have previously used this short term cytotoxicity assay and the relapse assay to assess silk hydrogels ${ }^{33}$ and films ${ }^{24}$ for their ability to release cytotoxic drugs and precision medicines ${ }^{13-14}$. In all these previous studies, we obtained a similar therapeutic response to that detailed here (Fig. 3).

Our previous excellent translation of in vitro results into orthotopic neuroblastoma and breast cancer in vivo models ${ }^{13-14,24}$ prompted us to assess the therapeutic potential of doxorubicinloaded PEG-heparin microparticle aggregates in orthotopic MDA-MB-231 breast cancers. Local injection of doxorubicin-loaded PEG-heparin microparticle aggregates, once breast tumours were established, revealed that focal doxorubicin delivery significantly reduced tumour growth from 3 weeks until the end of the study when compared to the bolus control treatment (Fig. 4b). We also observed significantly smaller primary tumours and reduced metastasis for animals that were treated with doxorubicin-loaded PEG-heparin hydrogel microparticle aggregates. However, none of the animals showed complete tumour regression, which was previously observed for doxorubicin-loaded silk hydrogels in 2 out of 5 animals ${ }^{33}$. Furthermore, the primary tumour sizes in the current study and in the silk hydrogel study were $345 \mathrm{mg}$ and $142 \mathrm{mg}$, respectively, although the tumour model, doxorubicin dose and dosing schedule were identical in both studies. The in vitro doxorubicin release from PEG-heparin hydrogel microparticle aggregates showed a substantially faster drug release profile when compared to silk hydrogels ${ }^{33}$. This is likely to have direct consequences in vivo. Therefore, additional refinements of anticancer drug release from 
PEG-heparin hydrogel microparticle aggregates is likely to result in further enhancement of the antitumour response.

Histological examination showed a good in vivo tissue compatibility of PEG-heparin hydrogel microparticle aggregates, which was coupled with the ability of these hydrogels to retain their shape at the injection site. This observation is in line with previous observations that PEG based hydrogels are particularly well suited for in vivo applications ${ }^{28}$. For example, previous in vivo studies with PEG-heparin hydrogels showed that application of these hydrogels into the striatum of rats induced a low transient presence of microglia and a mild astroglial reaction at the hydrogel-brain interface at one week after implantation ${ }^{22}$. Over the 21 day study period, a good histocompatibility was observed, with no significant adverse tissue responses ${ }^{22}$.

\section{Conclusions}

This study demonstrates that heparin-modified PEG hydrogel microparticle aggregates are well placed for the focal delivery of anticancer drugs. Doxorubicin-loaded hydrogel microparticle aggregates showed an excellent in vitro response and a significant antitumour effect in an aggressive orthotopic breast cancer model. Drug-loaded PEG-heparin hydrogel microparticle aggregates induced a reduction in both primary tumour growth and metastasis, while histological assessment at the end of the study showed good histocompatibility. Overall, these findings, when combined with prior in vivo data on PEG-heparin hydrogels, support a viable future for PEGheparin hydrogel microparticle aggregates in (anticancer) drug delivery applications.

\section{Author Contributions}


FPS initiated study. FPS and MT carried out experiments and analyzed the data. All authors (FPS, MT, UF, DLK and CW) designed research, discussed the results, and/or advised on the analysis. FPS wrote the manuscript with support from the other authors. All authors have given approval to the final version of the manuscript. $\ddagger$ These authors contributed equally (FPS and MT).

\section{Acknowledgements}

The authors thank Ilona Konrad for technical assistance with in vivo studies. This work was supported by project grants NIH/NIBIB grant P41 EB002520-05 (Tissue Engineering Resource Center) (DLK), a Marie Curie FP7 Career Integration Grant 334134 within the $7^{\text {th }}$ European Union Framework Program (FPS).

\section{Supporting Information}

The following files are available free of charge.

Supplementary Figure 1 Representative images of PEG-heparin hydrogel microparticle aggregates loaded with different amounts of doxorubicin.

Supplementary Figure 2. Drug release from PEG-heparin hydrogel microparticle aggregates (HydroMicroAgg).

\section{Abbreviations \\ DCIS, ductal carcinoma in situ}


EDC hydrochloride, $N$-(3-dimethylaminopropyl)-N'-ethylcarbodiimide hydrochloride

H\&E staining, Haematoxylin and Eosin staining

HydroMicroAgg, microparticle aggregates

MPP, matrix metalloprotease

MTT, (3-(4,5-dimethylthiazol-2-yl)-2,5-diphenyltetrazolium bromide

MW, molecular weight

NHS, $N$-hydroxysulfosuccinimide

PBS, phosphate buffered saline

PEG, polyethylene glycol

PHEMA, poly(2-hydroxyethyl methacrylate)

SD, standard deviation

solgel, solution-gel

starPEG, polyethylene glycol stars

\section{References}

1. Brem, H.; Piantadosi, S.; Burger, P. C.; Walker, M.; Selker, R.; Vick, N. A.; Black, K.; Sisti, M.; Brem, S.; Mohr, G.; et al., Placebo-controlled trial of safety and efficacy of intraoperative controlled delivery by biodegradable polymers of chemotherapy for recurrent gliomas. The Polymer-brain Tumor Treatment Group. Lancet 1995, 345 (8956), 1008-1012. DOI:10.1016/S0140-6736(95)90755-6

2. Tobias, J.; Hochhauser, D., Cancer and its management. 6 ed.; Wiley- Blackwell: Oxford, UK, 2010. 
3. Fisher, B.; Anderson, S.; Bryant, J.; Margolese, R. G.; Deutsch, M.; Fisher, E. R.; Jeong, J. H.; Wolmark, N., Twenty-year follow-up of a randomized trial comparing total mastectomy, lumpectomy, and lumpectomy plus irradiation for the treatment of invasive breast cancer. N. Engl. J. Med. 2002, 347, 1233-1241. DOI: 10.1056/NEJMoa022152.

4. Veronesi, U.; Cascinelli, N.; Mariani, L.; Greco, M.; Saccozzi, R.; Luini, A.; Aguilar, M.; Marubini, E., Twenty-year follow-up of a randomized study comparing breast-conserving surgery with radical mastectomy for early breast cancer. N. Engl. J. Med. 2002, 347, 1227 1232. DOI: 10.1056/NEJMoa020989.

5. Vaidya, J. S.; Joseph, D. J.; Tobias, J. S.; Bulsara, M.; Wenz, F.; Saunders, C.; Alvarado, M.; Flyger, H. L.; Massarut, S.; Eiermann, W.; Keshtgar, M.; Dewar, J.; KrausTiefenbacher, U.; Sutterlin, M.; Esserman, L.; Holtveg, H. M.; Roncadin, M.; Pigorsch, S.; Metaxas, M.; Falzon, M.; Matthews, A.; Corica, T.; Williams, N. R.; Baum, M., Targeted intraoperative radiotherapy versus whole breast radiotherapy for breast cancer (TARGIT-A trial): an international, prospective, randomised, non-inferiority phase 3 trial. Lancet $\mathbf{2 0 1 0}$, 376, 91-102. DOI: 10.1016/S0140-6736(10)60837-9.

6. Obeng-Gyasi, S.; Ong, C.; Hwang, E. S., Contemporary management of ductal carcinoma in situ and lobular carcinoma in situ. Chin. Clin. Oncol. 2016, 5, 32. DOI:

10.21037/cco.2016.04.02.

7. Nguyen, M. K.; Alsberg, E., Bioactive factor delivery strategies from engineered polymer hydrogels for therapeutic medicine. Prog. Polymer Sci. 2014, 39, 1235-1265.DOI:

10.1016/j.progpolymsci.2013.12.001 
8. Qiu, Y.; Park, K., Environment-sensitive hydrogels for drug delivery. Adv. Drug Deliver. Rev. 2012, 64, 49-60. DOI: Doi 10.1016/J.Addr.2012.09.024.

9. Xu, S.; Wang, W.; Li, X.; Liu, J.; Dong, A.; Deng, L., Sustained release of PTXincorporated nanoparticles synergized by burst release of $\mathrm{DOXHCl}$ from thermosensitive modified PEG/PCL hydrogel to improve anti-tumor efficiency. Eur. J. Pharm. Sci. 2014, 62, 267-273. DOI: 10.1016/j.ejps.2014.06.002.

10. Ashley, G. W.; Henise, J.; Reid, R.; Santi, D. V., Hydrogel drug delivery system with predictable and tunable drug release and degradation rates. Proc. Natl. Acad. Sci. USA 2013, 110, 2318-2323. DOI: 10.1073/pnas.1215498110.

11. Yu, J.; Xu, X.; Yao, F.; Luo, Z.; Jin, L.; Xie, B.; Shi, S.; Ma, H.; Li, X.; Chen, H., In situ covalently cross-linked PEG hydrogel for ocular drug delivery applications. Int. J. Pharm. 2014, 470, 151-157. DOI: 10.1016/j.ijpharm.2014.04.053.

12. Marques, J. G.; Gaspar, V. M.; Markl, D.; Costa, E. C.; Gallardo, E.; Correia, I. J., Codelivery of Sildenafil (Viagra((R))) and Crizotinib for synergistic and improved anti-tumoral therapy. Pharm. Res. 2014, 31, 2516-1528. DOI: 10.1007/s11095-014-1347-x.

13. Seib, F. P.; Coburn, J.; Konrad, I.; Klebanov, N.; Jones, G. T.; Blackwood, B.; Charest, A.; Kaplan, D. L.; Chiu, B., Focal therapy of neuroblastoma using silk films to deliver kinase and chemotherapeutic agents in vivo. Acta Biomater. 2015, 20, 32-38. DOI: 10.1016/j.actbio.2015.04.003.

14. Seib, F. P.; Kaplan, D. L., Silk for Drug Delivery Applications: Opportunities and Challenges. Isr. J. of Chem. 2013, 53, 756-766. DOI: Doi 10.1002/Ijch.201300083. 
15. Maitz, M. F.; Freudenberg, U.; Tsurkan, M. V.; Fischer, M.; Beyrich, T.; Werner, C., Bio-responsive polymer hydrogels homeostatically regulate blood coagulation. Nat. Commun. 2013, 4, 2168. DOI: $10.1038 /$ ncomms3168.

16. Mousa, S. A.; Petersen, L. J., Anti-cancer properties of low-molecular-weight heparin: preclinical evidence. Thromb. Haemost. 2009, 102, 258-267. DOI: 10.1160/TH08-12-0832.

17. Niers, T. M.; Klerk, C. P.; DiNisio, M.; Van Noorden, C. J.; Buller, H. R.; Reitsma, P. H.; Richel, D. J., Mechanisms of heparin induced anti-cancer activity in experimental cancer models. Crit. Rev. Oncol. Hematol. 2007, 61, 195-207. DOI:

10.1016/j.critrevonc.2006.07.007.

18. Smorenburg, S. M.; Vink, R.; Otten, H. M.; Swaneveld, F.; Buller, H. R., The effects of vitamin K-antagonists on survival of patients with malignancy: a systematic analysis.

Thromb. Haemost. 2001, 86, 1586-1587.

19. Rohloff, J.; Zinke, J.; Schoppmeyer, K.; Tannapfel, A.; Witzigmann, H.; Mossner, J.; Wittekind, C.; Caca, K., Heparanase expression is a prognostic indicator for postoperative survival in pancreatic adenocarcinoma. Br. J. Cancer 2002, 86, 1270-1275. DOI: 10.1038/sj.bjc.6600232.

20. Chwalek, K.; Tsurkan, M. V.; Freudenberg, U.; Werner, C., Glycosaminoglycan-based hydrogels to modulate heterocellular communication in in vitro angiogenesis models. Sci. Rep. 2014, 4, 4414. DOI: 10.1038/srep04414. 
21. Tsurkan, M. V.; Chwalek, K.; Prokoph, S.; Zieris, A.; Levental, K. R.; Freudenberg, U.; Werner, C., Defined polymer-peptide conjugates to form cell-instructive starPEG-heparin matrices in situ. Adv. Mater. 2013, 25, 2606-2610. DOI: 10.1002/adma.201300691.

22. Freudenberg, U.; Hermann, A.; Welzel, P. B.; Stirl, K.; Schwarz, S. C.; Grimmer, M.; Zieris, A.; Panyanuwat, W.; Zschoche, S.; Meinhold, D.; Storch, A.; Werner, C., A starPEG-heparin hydrogel platform to aid cell replacement therapies for neurodegenerative diseases. Biomaterials 2009, 30, 5049-5060. DOI: 10.1016/j.biomaterials.2009.06.002.

23. Tsurkan, M. V.; Hauser, P. V.; Zieris, A.; Carvalhosa, R.; Bussolati, B.; Freudenberg, U.; Camussi, G.; Werner, C., Growth factor delivery from hydrogel particle aggregates to promote tubular regeneration after acute kidney injury. J. Control. Release 2013, 167, 248255. DOI: 10.1016/j.jconrel.2013.01.030.

24. Seib, F. P.; Kaplan, D. L., Doxorubicin-loaded silk films: Drug-silk interactions and in vivo performance in human orthotopic breast cancer. Biomaterials 2012, 33, 8442-8450. DOI: Doi 10.1016/J.Biomaterials.2012.08.004.

25. Goldstein, R. H.; Reagan, M. R.; Anderson, K.; Kaplan, D. L.; Rosenblatt, M., Human bone marrow-derived MSCs can home to orthotopic breast cancer tumors and promote bone metastasis. Cancer Res. 2010, 70, 10044-10050. DOI: 10.1158/0008-5472.CAN-10-1254.

26. Duncan, R.; Gaspar, R., Nanomedicine(s) under the microscope. Mol. Pharm. 2011, 8 , 2101-2141. DOI: 10.1021/mp200394t. 
27. Pasut, G.; Veronese, F. M., State of the art in PEGylation: the great versatility achieved after forty years of research. J. Control. Release 2012, 161, 461-472. DOI:

10.1016/j.jconrel.2011.10.037.

28. Ratner, B. D., Reducing capsular thickness and enhancing angiogenesis around implant drug release systems. J Control Release 2002, 78, 211-218. DOI: 10.1016/S0168$3659(01) 00502-8$

29. Franz, S.; Rammelt, S.; Scharnweber, D.; Simon, J. C., Immune responses to implants a review of the implications for the design of immunomodulatory biomaterials. Biomaterials 2011, 32 (28), 6692-6709. DOI: 10.1016/j.biomaterials.2011.05.078.

30. Zhang, L.; Cao, Z.; Bai, T.; Carr, L.; Ella-Menye, J. R.; Irvin, C.; Ratner, B. D.; Jiang, S., Zwitterionic hydrogels implanted in mice resist the foreign-body reaction. Nat. Biotechnol. 2013, 31, 553-556. DOI: 10.1038/nbt.2580.

31. Tsurkan, M. V.; Levental, K. R.; Freudenberg, U.; Werner, C., Enzymatically degradable heparin-polyethylene glycol gels with controlled mechanical properties. Chem. Commun. (Camb) 2010, 46, 1141-1143. DOI: 10.1039/b921616b.

32. Tsurkan, M. V.; Chwalek, K.; Schoder, M.; Freudenberg, U.; Werner, C., Chemoselective peptide functionalization of starPEG-GAG hydrogels. Bioconjug. Chem. 2014, 25, 1942-1950. DOI: 10.1021/bc500217z.

33. Seib, F. P.; Pritchard, E. M.; Kaplan, D. L., Self-Assembling Doxorubicin Silk Hydrogels for the Focal Treatment of Primary Breast Cancer. Adv. Funct. Mater. 2013, 23, 58-65. DOI: Doi 10.1002/Adfm.201201238. 


\title{
Heparin modified polyethylene glycol
}

\section{microparticle aggregates for focal cancer}

\author{
chemotherapy
}

F. Philipp Seib, Mikhail Tsurkan, Uwe Freudenberg, David L. Kaplan, Carsten Werner

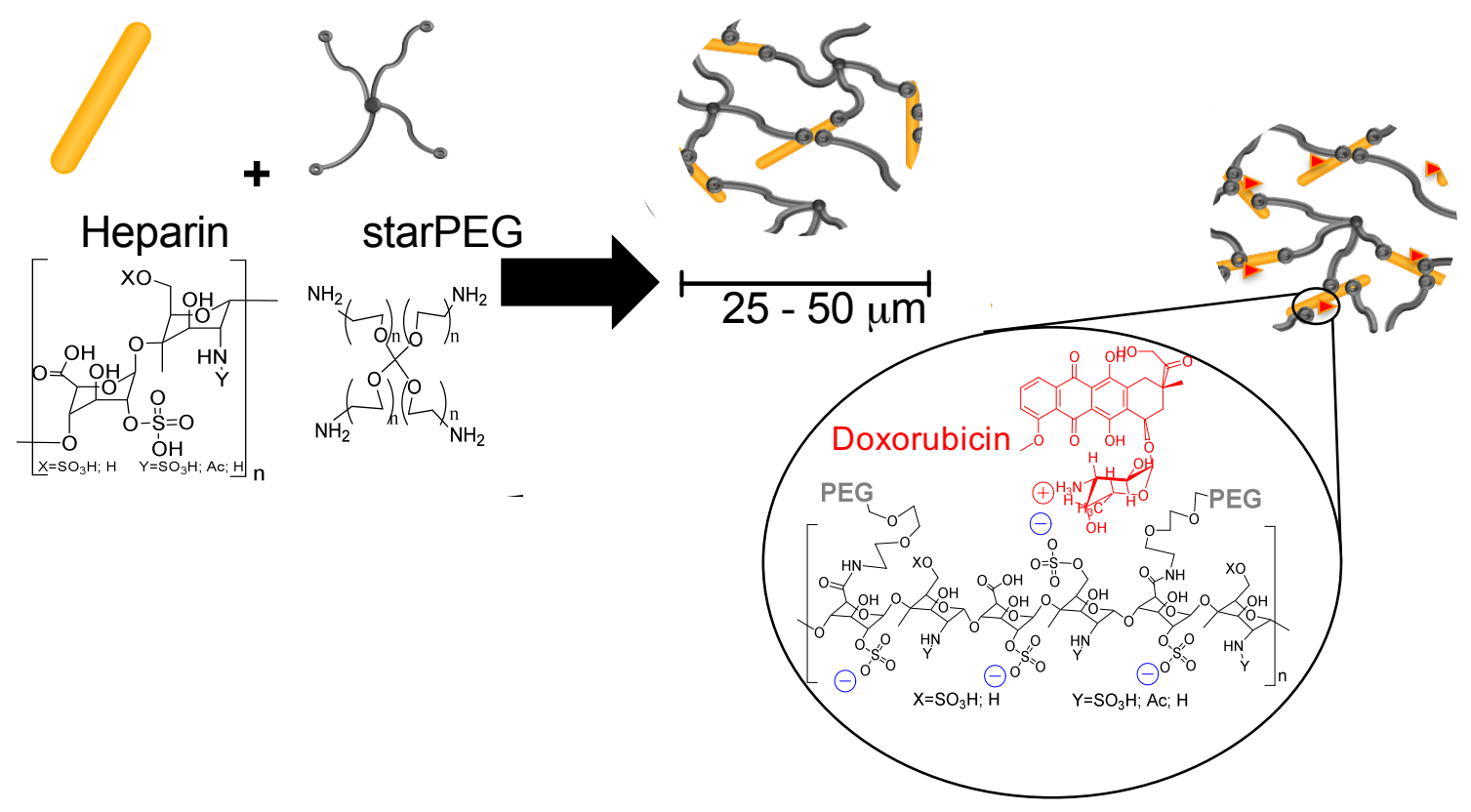

Table of content graphic 


\title{
Heparin modified polyethylene glycol
}

\section{microparticle aggregates for focal cancer}

\author{
chemotherapy
}

F. Philipp Seib ${ }^{1,2, \#}$, Mikhail Tsurkan ${ }^{2, \#}$, Uwe Freudenberg ${ }^{2}$, David L. Kaplan ${ }^{3}$, Carsten

Werner $^{2, *}$

\section{Supplementary information}

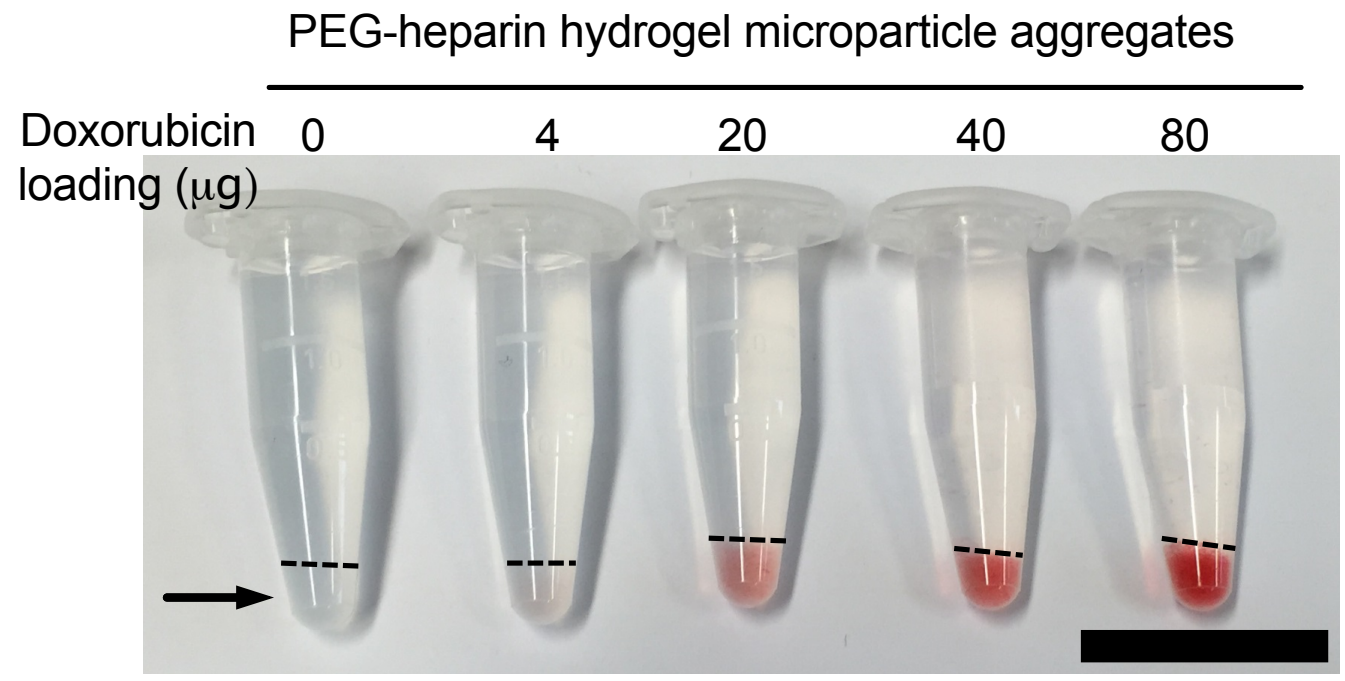

Supplementary Figure 1 Representative images of PEG-heparin hydrogel microparticle aggregates loaded with different amounts of doxorubicin. Dashed line indicates the interface between the hydrogel (arrow) and PBS; scale bare $15 \mathrm{~mm}$. 
A

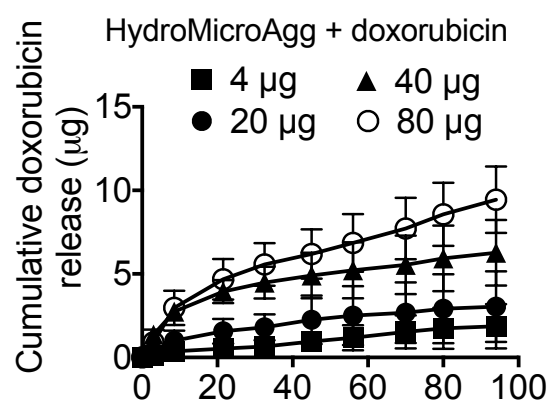

C

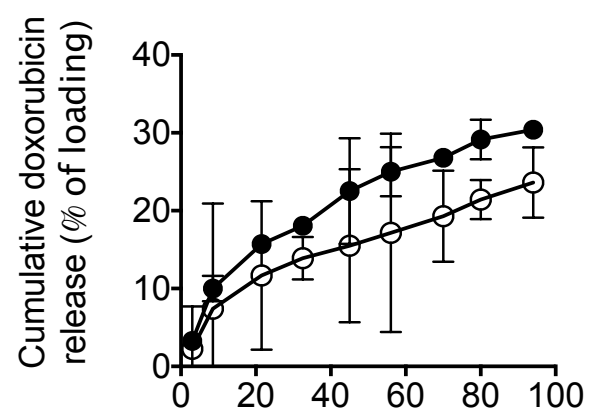

$\mathbf{E}$

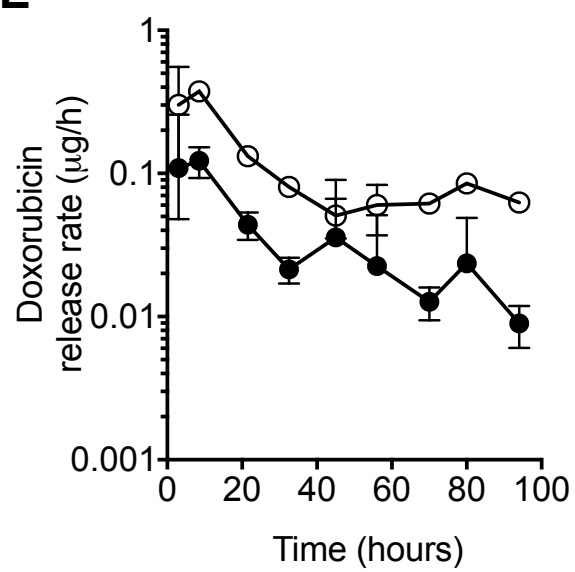

B

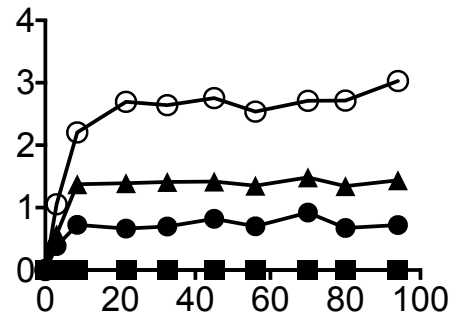

D

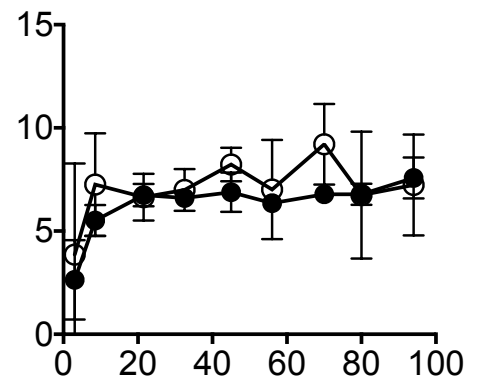

$\mathbf{F}$

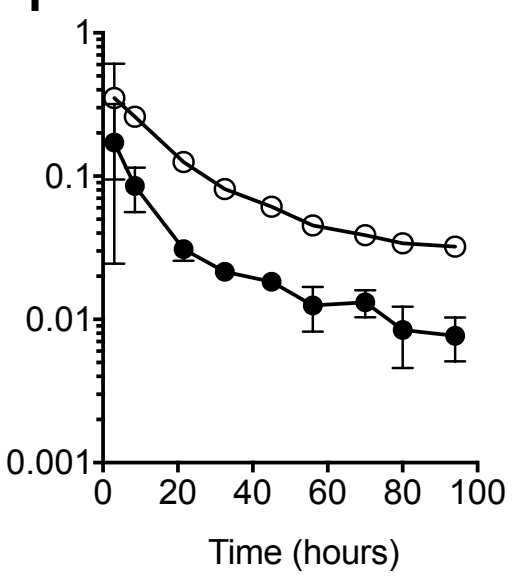

Supplementary Figure 2. Drug release from PEG-heparin hydrogel microparticle aggregates (HydroMicroAgg). Cumulative doxorubicin release when buffer was (A) replaced with fresh one at each sampling time point or (B) kept throughout the experiment. Corresponding doxorubicin release relative to initial loading. Doxorubicin release rates when the release buffer was replaced 
(E) or (F) kept. Note that release was from $50 \mu 1$ HydroMicroAgg samples; nominal doxorubicin loadings of $4-80 \mu \mathrm{g}$ correspond to $100 \mu \mathrm{l}$ of sample 